\title{
Balkanologie
}

Balkanologie Revue d'études pluridisciplinaires

Vol. II, nº 1 | 1998

Volume II Numéro 1

\section{La « revanche des campagnes »}

Entre réalité sociologique et mythe nationaliste

Xavier Bougarel

\section{CpenEdition}

Journals

Édition électronique

URL : http://journals.openedition.org/balkanologie/237

DOI : 10.4000/balkanologie.237

ISSN : 1965-0582

Éditeur

Association française d'études sur les Balkans (Afebalk)

Édition imprimée

Date de publication : 1 juillet 1998

ISSN : 1279-7952

Référence électronique

Xavier Bougarel, « La « revanche des campagnes » », Balkanologie [En ligne], Vol. II, n 1 | 1998, mis en ligne le 02 juin 2008, consulté le 17 décembre 2020. URL : http://journals.openedition.org/ balkanologie/237 ; DOI : https://doi.org/10.4000/balkanologie.237

Ce document a été généré automatiquement le 17 décembre 2020.

(c) Tous droits réservés 


\title{
La « revanche des campagnes »
}

\author{
Entre réalité sociologique et mythe nationaliste
}

\author{
Xavier Bougarel
}

\section{Les conflits yougoslaves comme « revanche des campagnes »}

1 Nombreux sont les journalistes et les intellectuels qui, à juste titre, ont refusé une présentation hobbesienne des conflits yougoslaves, l'idée d'une " guerre de tous contre tous ", reflet de la haine séculaire que se voueraient des peuples balkaniques plus ou moins barbares. Hélas, nombre d'entre eux ont réintroduit par la fenêtre ce qu'ils venaient de chasser par la porte. La présentation des conflits yougoslaves et des phénomènes miliciens qui les ont caractérisés en termes de «revanche des campagnes ", en particulier, a donné lieu à bien des dérapages. Ainsi, dans son ouvrage intitulé Sarajevo, l'architecte et les barbares, l'architecte bosniaque Ivan Straus parle de "primitifs armés", "édentés et mal lavés " à propos des combattants serbes qui entourent et bombardent la ville, et des «nouveaux venus, à peine cultivés ", pour qualifier les milieux dont ils sont issus ${ }^{1}$. Si de tels propos peuvent s'expliquer, venant d'une personne directement affectée par la guerre, il est regrettable qu'ils aient été élevés au rang de catégories d'analyse par certains commentateurs occidentaux.

2 L'identification des miliciens à des ruraux incultes et barbares n'est pas le fait des seuls intellectuels pacifistes. À Sarajevo, la population opposait volontiers le raja, petit peuple pacifique de la ville, aux papci belliqueux des collines ${ }^{2}$. Cette même hostilité se retrouvait aussi dans la bouche des combattants, pour qualifier l'adversaire : chez les Serbes, les Musulmans étaient souvent traités de Balije; inversement, les Musulmans parlaient des Vlasi («Valaques») pour désigner les Serbes. Dans un cas comme dans l'autre, l'adversaire était assimilé à une population nomade, étrangère à la polis et à la civilisation ${ }^{3}$. L'idée d'une ville "civilisée" opposée à une campagne "barbare", sujet de conversation privilégié entre les intellectuels locaux et leurs visiteurs étrangers, savait aussi nourrir les haines de classe et les discours guerriers. 
Il ne s'agit pas ici de nier les liens qui associent les phénomènes miliciens aux régions rurales et montagnardes de l'espace yougoslave. De fait, bon nombre des entrepreneurs miliciens sont originaires des Alpes dinariques ${ }^{4}$, et les populations de ces régions constituent généralement les premiers bastions des mobilisations nationalistes, politiques puis miliciennes. Inversement, les conflits yougoslaves sont marqués par un acharnement particulier contre les centres urbains et les symboles architecturaux de leur urbanité, comme le montrent la destruction délibérée de Vukovar en Croatie, ou les sièges de Sarajevo et de Mostar en Bosnie-Herzégovine.

4 Mais il suffit de voir l'état de dévastation de nombreux villages en Croatie et en BosnieHerzégovine, n'ayant rien à envier à celui de Vukovar ou de Mostar, pour contaster à quel point l'idée d'une "revanche des campagnes" est déplacée dans le cas des conflits yougoslaves. Si les rapports entre ville et campagne occupent une place centrale dans ces conflits comme dans le discours de leurs protagonistes, encore faut-il savoir quelle est la part de la réalité et la part du mythe, quelle est la véritable signification de ces rapports, et se demander en particulier ce qu'ils révèlent (ou dissimulent) sur les rapports entre milices, État et société dans l'espace yougoslave.

\section{Dinko Tomašić et la culture clanique}

5 L'insistance sur l'opposition entre ville et campagne, ou plus précisément entre ville, plaine et montagne, est une constante dans les travaux sociologiques et ethnologiques consacrés aux Balkans, et ce depuis l'ouvrage fondateur du géographe serbe Jovan Cvijić (1865-1927), La péninsule balkanique, publié au début du siècle ${ }^{5}$. Il n'est donc pas surprenant de retrouver cette articulation dans l'analyse des conflits yougoslaves.

6 Une bonne illustration de ce phénomène est l'engouement perceptible en Croatie pour le sociologue Dinko Tomašić (1902-1975), dont les principaux travaux, publiés dans les années trente et quarante, insistent sur l'opposition de deux "types culturels" au sein des sociétés balkaniques : la culture clanique (plemenska kultura) d'une part, organisée autour du pleme (clan, tribu) et caratéristique des populations pastorales de la montagne, et la culture coopérative (zadružna kultura) d'autre part, organisée autour de la zadruga (famille élargie constituée en entité économique indivise), et caractéristique des populations agricoles des plaines.

7 Dans un article intitulé «La culture clanique et ses traces contemporaines », et publié en $1936^{6}$, D. Tomašić s'intéresse plus spécifiquement à la place de la culture clanique dans les pratiques de la violence des sociétés balkaniques, et dans la formation des États nationaux dans les Balkans. D'après lui, la force physique et la mobilité géographique des populations dinariques expliquent que «l'organisation sociale clanique ait donné naissance aux bandes [čete] de pillards et de guerriers, qu'en son sein le port d'arme soit devenu synonyme d'humanité, et que la valeur sociale dominante y soit l'“héroïsme", c'est-à-dire la surenchère dans la rapine et dans le crime ». De la culture patriarcale et clanique des populations dinariques émergerait donc un culte de la violence centrée autour de la figure mythique des hajduk et des uskok (bandits sociaux), qui ne tarderait pas à dominer les États et les sociétés balkaniques.

8 D'après D. Tomašić toujours, les hajduk, dont « le seul but est le pillage et la rapine », se laissent néammoins utiliser comme «porteurs d'idéologie et fondateurs d'État » par les puissances européennes opposées à l'Empire ottoman : « les bandes de hajduk reçoivent 
un étendard marqué d'une croix, et les harambaša [chefs de bande] un soutien moral et matériel pour déclencher avec leur bande des soulèvements de "libération" ". Monopolisant alors l'État et son appareil militaire, les populations dinariques s'appuient également sur leur art de la contrebande pour s'emparer des activités commerciales, et imposent finalement leur domination économique et culturelle à la paysannerie des plaines comme à la bourgeoisie des villes. D. Tomašić voit dans cette domination de la culture clanique la cause du caractère autoritaire et instable des États balkaniques apparus au XIX ${ }^{\text {ème }}$ siècle, et tente ainsi d'expliquer la prédominance serbe dans la première Yougoslavie, l'émergence du mouvement des partisans pendant la Seconde Guerre mondiale, ou encore, en élargissant son champ d'investigation, la victoire du communisme en Russie et en Europe de l'est ${ }^{7}$.

9 Directement confrontés à la violence de la guerre, certains sociologues croates ont redécouvert les thèses de D. Tomašić. Dès 1992, Aleksandar Stulhofer publie dans la revue Društvena istraživanja ( Recherches sociales ») un article intitulé « Une prévision de la guerre ? L'ethno-sociologie de Dinko Tomašić »" ${ }^{8}$. Quelques mois plus tard, cette même revue de sociologie consacre un numéro spécial à ce même auteur' ${ }^{9}$. Enfin, en 1993, trois sociologues croates (Stjepan Meštrović, Slaven Letica et Miroslav Goreta) publient aux États-Unis un livre intitulé Habits of the Balkan Heart, et consacré à l'influence des mentalités (social characters) sur les formes du post-communisme en Europe centrale et orientale ${ }^{10}$. S'intéressant aux conflits yougoslaves, ces trois auteurs recourent abondamment aux travaux de D. Tomašić, et opposent à leur tour la mentalité cosmopolite des villes et celle, pacifique, des plaines, à une mentalité dinarique "assoiffée de pouvoir et aggressive», incarnée par les Serbes et les Monténégrins. Dès lors, l'agression serbe contre la Croatie n'a plus de secret : «il est bien connu en Yougoslavie que les Serbes et les Monténégrins adhèrent à une sorte de culte du guerrier. Ils ont continuellement dominé la police et les forces armées. La possession traditionnelle des armes et la pratique de la chasse participent d'un système de valeurs machiste. À l'intérieur de la Yougoslavie, ils sont connus pour être bornés, irascibles et émotionnellement instables ».

\section{Réalité sociologique ou mythe nationaliste?}

10 Identifiant la mentalité dinarique aux seules populations orthodoxes, S. Meštrović, S. Letica et $\mathrm{M}$. Goreta superposent à ce conflit de mentalités un affrontement entre cultures, rappelant que « la ligne de partage entre l'est et l'ouest passe à peu près le long de la frontière actuelle entre la Croatie et la Serbie, connue sous le nom de région de la Krajina », et que cette région est celle "dans laquelle se sont déroulés les combats les plus féroces en 1991, et lors des précédentes guerres balkaniques ». Pour eux, le conflit en Croatie se résume à l'affrontement entre deux aires culturelles, l'une, occidentale et catholique, étant représentée par les Croates et les Slovènes, et l'autre, orientale et orthodoxe, par les Serbes et les Monténégrins :

L'Église catholique a maintenu un fondement culturel universaliste qui, resté médiéval par bien des aspects, n'en est pas moins typiquement occidental. C'est pourquoi la Croatie et la Slovénie ont conduit la rébellion anti-communiste en 1989, ont voté pour la démocratie, tendent à favoriser le pluralisme, veulent rejoindre la Communauté Européenne (...). Par contraste, la Serbie et le Monténégro se tournent vers la sphère culturelle orientale. Ils ont des liens étroits avec la Russie et sont néo-communistes, suite à des élections au cours desquelles la démocratie a été 
rejetée. D'orientation religieuse orthodoxe ou musulmane, ils aspirent à une structure pyramidale du pouvoir et sont militaristes.

11 Une telle présentation de la crise yougoslave, dans laquelle la Bosnie-Herzégovine disparaît comme par enchantement (la Krajina se situe en fait à la frontière croatobosniaque), apparaît pour le moins simpliste. Ses présupposés culturalistes, reflets des analyses du post-communisme opposant une Europe centrale démocratique à une Europe orientale autoritaire ou chaotique, ont déjà fait l'objet de critiques pertinentes qu'il est inutile de reprendre $\mathrm{ici}^{11}$. Il faut par contre insister sur le fait que les populations dinariques ne sont pas exclusivement serbes, et que le lien étroit qui unit ces populations aux phénomènes miliciens n'est pas propre au camp serbe, comme en témoignent le rôle des Croates d'Herzégovine en Slavonie orientale, ou celui des Musulmans du Sandžak à Sarajevo. De ce point de vue, quand S. Meštrović, S. Letica et M. Goreta placent la frontière entre cultures occidentale et orientale à Međugorje, lieu de pélerinage catholique situé en Herzégovine occidentale, c'est-à-dire au coeur du principal "fief" du HVO croate, cela ne manque pas de faire sourire ${ }^{12}$ !

Cette "ethnicisation" abusive de la réalité sociale et culturelle des sociétés balkaniques n'est pas récente : elle se retrouve chez $\mathrm{D}$. Tomašić et ses prédécesseurs. Les "types culturels" de D. Tomašić, en effet, ne sont qu'une reprise des "types psychologiques" esquissés par Jovan Cvijić dans La péninsule balkanique. Pour ce dernier toutefois, le "régime patriarcal" caractéristique du "type dinarique" n'est pas un état inférieur de la civilisation, mais au contraire son facteur régénérant, face à la raya, "classe inférieure et servile » des plaines, et à la population urbaine, qui « dégénère (...) et s'abâtardit au bout de quelques générations ». Et J. Cvijić d'en appeler alors aux vertus militaires de la population dinarique, incarnée par «les hajduk, les uskok, les vengeurs", pour conquérir « la liberté et l'indépendance de toutes les contrées qu'[elle] sait, d'après la tradition, avoir fait partie de son État [serbe], et qui sont habités par la pauvre raya, par les hommes oppressés de sa race ».

13 Au prédateur de D. Tomašić s'oppose donc le libérateur de J. Cvijić. Mais, dans les deux cas, les rapports entre ville, plaine et montagne sont réinterprétés en termes ethniques et nationaux. Certes, ces deux interprétations renvoient à des réalités historiques fortes de l'espace yougoslave: dans l'Empire ottoman comme dans l'Empire austro-hongrois, statut social, localisation géographique et appartenance ethnique sont indéniablement liés. Mais elles en constituent surtout des reconstructions idéologiques, et doivent être à ce titre rattachées aux différents nationalismes qui s'affrontent dans ce même espace. Du reste, J. Cvijić n'est-il pas un des idéologues du nationalisme grand-serbe, et D. Tomašić un intellectuel proche du Parti paysan croate? Et est-ce un hasard si la cristallisation du nationalisme musulman au cours du conflit en Bosnie-Herzégovine va de pair avec l'émergence d'un discours voyant dans celui-ci un affrontement entre la « civilisation urbaine » des Musulmans et l'» âme barbare et tribale » des Serbes ${ }^{13}$, et opposant «la zadruga slave, rurale et patriarcale » à l'»ancien čitluk bosniaque ", présenté comme une institution citadine ${ }^{14}$ ?

\section{État de nature hobbesien ou assabiyyas khalduniennes?}

14 Se focalisant sur ces populations dinariques «qui ont pris le contrôle des espaces urbains et ont appris à consolider leur pouvoir en propageant une idéologie religieuse, 
nationale ou partisane ", D. Tomašić réduit l'histoire des sociétés balkaniques à une récurrence tragique des "tensions entre paysan, berger et citadin $»^{15}$. Cette vision cyclique de l'histoire n'est pas sans affinités avec celle d'Ibn Khaldun, souvent mise en avant dans l'étude des sociétés et des États du monde arabo-musulman : le pleme évoque irrésistiblement l'assabiyya khaldunienne (groupe de solidarité fondé sur des liens de sang ou d'allégeance), et l'opposition entre ville, plaine et montagne rappelle celle établie par Ibn Khaldun entre badawa (nomadisme) et hadara (urbanité) ${ }^{16}$.

Chez Ibn Khaldun toutefois, l'assabiyya apparaît plutôt un facteur régénérant de l'État : sur ce point, J. Cvijić est probablement plus khaldunien que D. Tomašić. Autre différence, plus fondamentale, J. Cvijić comme D. Tomašić tendent à définir le pleme comme préexistant et extérieur à l'État, et ce dernier comme un simple instrument dans les rapports entre ville, plaine et montagne. Chez Ibn Khaldun au contraire, l'État (dawla) se situe au centre des rapports entre badawa et hadara, et constitue l'enjeu central de l'action des assabiyya. Comme l'écrit Aziz Al-Azmeh, dans la pensée khaldunienne, " sans la dawla [l'État], le concept d'assabiyya serait superflu, nonobstant son "existence réelle". En tant que concept, il est sujet aux exigences du concept d'État et ne peut exister sans lui, ni, en fait, sans rester dans sa proximité ${ }^{17}$.

Il est donc peut-être nécessaire, pour comprendre les dynamiques profondes des conflits yougoslaves, de replacer l'État au centre de leur problématique. Au demeurant, et contrairement à ce que sous-entendent les présentations hobbesiennes (avouées ou honteuses) de ces conflits, la réalité des populations dinariques, leur organisation clanique et leur culture de la violence, ne peuvent être appréhendées sans tenir compte de leur rapport à l'État.

\section{L'État et le hajduk : une culture de confins}

Dans l'histoire de l'espace yougoslave, la montagne représente d'abord un refuge face aux Empires, comme le montrent l'autonomie de fait dont bénéficie le Monténégro au sein de l'Empire ottoman, ou la prédominance des populations serbe et croate en Herzégovine, région difficile d'accès et de ce fait peu touchée par l'islamisation. Mais elle représente aussi une position stratégique, un confin à tenir, comme l'illustrent le cas des populations musulmanes de Cazinska Krajina (région de Bihać), regroupées aux confins septentrionaux de l'Empire ottoman après s'être retirées de Slavonie et de Dalmatie, ou celui des populations serbes de Krajina, installées aux confins de l'Empire austro-hongrois après avoir fui l'Empire ottoman. Cette marque impériale sur la géographie des populations dinariques se reflète aujourd'hui encore dans leur situation périphérique au sein de leur propre communauté (Serbes de Krajina, Croates d'Herzégovine, Musulmans du Sandžak, etc.).

Redisposant les populations de l'espace yougoslave, les Empires savent aussi en instrumentaliser les modes d'organisation sociale et les pratiques de la violence. Certes, pendant toute la période ottomane, la force des structures claniques au Monténégro explique sa capacité de résistance au pouvoir central, et s'accompagne de raids et de pillages dans les provinces voisines. Mais l'expansion de l'Empire ottoman fait aussi appel à ces structures claniques et à ces pratiques de prédation, comme l'illustre aux $\mathrm{XV}^{\text {ème }}$ et XVI ${ }^{\mathrm{èm}}$ siècles le cas des martolos et des vojnuk, troupes auxiliaires recrutées au sein des populations nomades chrétiennes des Balkans. De même, l'Empire ottoman 
s'appuie, pour contrôler et taxer les populations conquises, sur leurs structures familiales, claniques et villageoises, contribuant ainsi à les pérenniser.

Cette articulation entre centre et périphérie se retrouve dans l'organisation militaire de l'Empire ottoman. Ses forces centrales sont constituées par les janissaires, arrachés à la périphérie par le système du devširme (levée d'enfants issus des populations chrétiennes), éduqués à Istambul et rattachés à la Porte par le versement d'une solde, alors que les sipahi, chargés de l'organisation des forces armées provinciales, sont rétribués par l'attribution d'un timar (concession foncière et fiscale). À partir du XVII ${ }^{\text {ème }}$ siècle, suite aux pertes territoriales sanctionnées par le traité de Karlowitz (1699), le système timariote se transforme en Bosnie-Herzégovine en un véritable système de confins militaires, les kapetanje (capitaineries), dont les prérogatives militaires et juridiques étendues expliquent la puissance des ayan (notables) bosniaques ${ }^{18}$.

À la même époque, l'Empire austro-hongrois établit son propre système de confins. Celui-ci diffère sensiblement du système ottoman, dans la mesure où il n'est pas fondé sur une aristocratie guerrière, mais sur une masse de paysans-soldats. L'Empire austrohongrois, en effet, accorde aux territoires nouvellement acquis un statut spécifique de Militärgrenzen (frontières militaires), et en confie la garde aux populations serbes venues de l'Empire ottoman, s'appuyant à cette fin sur des zadruga renforcées dans leurs fonctions économiques et militaires ${ }^{19}$. C'est dans ce cadre qu'il convient d'examiner les rapports complexes qui s'établissent entre les appareils militaires des Empires d'une part, les hajduk des régions dinariques d'autre part.

21 Hajduk et uskok désignent un seul et même phénomène, central dans l'histoire de l'espace yougoslave: le banditisme social. Les hajduk, souvent d'anciens kmet (serfs) ayant fui la pression fiscale des autorités ottomanes ou austro-hongroises, se retirent dans les régions montagneuses et s'y organisent en bandes armées (čete) commandées par un "chef de guerre" (vojvoda). Réfugiés dans les régions frontalières échappant au contrôle des autorités impériales, ils peuvent "sauter" d'un Empire à l'autre pour se livrer à la contrebande et au pillage, d'où le nom d'uskok qui leur est attribué dans certaines régions de l'espace yougoslave (skok signifie "saut" en serbo-croate). Les hajduk représentent donc une forme répandue de violence contre l'État, et occupent à ce titre une place centrale dans la tradition épique des populations dinariques.

Pourtant, dans la réalité, les rapports que les hajduk et les uskok entretiennent avec les Empires ne sont pas dénués d'ambiguïté. Dans les régions frontalières ou à l'occasion de campagnes militaires, ils sont recrutés dans des formations auxiliaires, appelées deli ("fous") du côté ottoman, pandur ou Freikörper ("corps-francs") du côté austro-hongrois, et dont l'engagement est rémunéré par une prédation directe (partage du butin) ou indirecte (attribution d'une terre). Cette intégration des hajduk dans les appareils militaires est attestée par le réemploi des termes hajduk et vojvoda dans certains statuts particuliers accordés aux populations serbes des Militärgrenzen.

Situé aux confins de deux Empires, l'espace yougoslave est donc marqué depuis longtemps par d'innombrables passages entre rébellion armée contre l'État et service armé de l'État, et D. Tomašić lui-même constate que

les régions frontalières sont particulièrement favorables à l'activité des hajduk, les régions où s'affrontent les sphères d'intérêt de deux ou plusieurs pouvoirs légaux et où l'administration est mal organisée (...). Si ces régions sont peuplées de représentants de la culture clanique ou nomade, encline au pillage et au rapt, le ralliement aux hajduk et le franchissement des frontières deviennent des 
phénomènes courants. D’un autre côté, ceux-ci seront tolérés, voire encouragés par les pouvoirs légaux environnants s'ils se trouvent en situation d'affrontement ouvert ou latent, afin de ruiner l'autorité du pouvoir situé de l'autre côté de la frontière, et faciliter ainsi d'éventuelles opérations militaires ${ }^{20}$. laquelle l'État est loin d'être absent. Du reste, l'appellation régionale de Krajina, érigée en barrière culturelle par S. Meštrović, S. Letica et M. Goreta, se retrouve en fait des deux côtés de la frontière croato-bosniaque (Kninska Krajina -Krajina de Knin- en Croatie, Cazinska Krajina -Krajina de Cazin- et bosanska Krajina -Krajina bosniaque- en Bosnie-Herzégovine), et provient du terme vojna krajina, qui signifie en serbo-croate... confin militaire.

\section{Tradition, modernisation et « retraditionnalisation »}

Quand D. Tomašić considère que «l'origine de ces États [nationaux des Balkans] peut être trouvée dans la personnalité des bergers prédateurs, dont l'appétit de pouvoir est resté pratiquement inchangé depuis des siècles $»^{21}$, il ne fait pas seulement l'impasse sur les rapports complexes qui, de longue date, associent culture clanique et formations étatiques dans les Balkans. Il omet également de prendre en compte les processus de modernisation socio-économique, politique et culturelle auxquels cette région du monde n'a évidemment pas échappé. De même, les analyses des phénomènes miliciens contemporains en termes de "mentalité dinarique" ou de "revanche des campagnes" font généralement l'impasse sur la modernisation extrêmement rapide qu'a connu l'espace yougoslave après 1945, en ignorent délibérément les déséquilibres et les contradictions, refusent, pour ainsi dire, de préciser quelle était la revanche à prendre.

Il apparaît pourtant difficile d'opposer sans réserve une culture urbaine à une ou plusieurs cultures rurales, dans un espace yougoslave où la part de la population vivant de l'agriculture est passée, en quelques décennies, de $73 \%$ (1948) à $27 \%$ (1981) de la population totale. 40 ans de modernisation et d'urbanisation accélérées ont déplacé les antagonismes traditionnels entre ville et campagne au sein même des villes, et menacé leurs équilibres internes en même temps qu'ils brisaient ceux de la société villageoise. C'est sans doute ce qui explique pourquoi des villes comme Sarajevo ou Mostar se sont trouvées au centre des affrontements entre 1992 et 1995, alors qu'elles étaient restées relativement épargnées par les combats de la Seconde Guerre mondiale, et pourquoi les mobilisations miliciennes y ont d'abord été le fait de populations néo-urbaines, mal intégrées à l'économie et à la culture urbaines, quand les élites urbaines représentaient le principal soutien des mouvements pacifistes.

À partir des années 1960, les déséquilibres de la modernisation de la société yougoslave ont provoqué sa "retraditionnalisation", reflétée en particulier par le renouveau des idéologies nationalistes et des pratiques communautaristes dans la vie politique yougoslave, et par une réactivation des solidarités familiales et claniques ${ }^{22}$. Les populations dinariques, marginalisées par la modernisation économique et situées en dehors des républiques correspondant à leur appartenance nationale, se sont les premières appuyées sur ces solidarités pour garder le contrôle de certains secteurs de l'appareil d'État (poids des Serbes de Krajina dans la police croate), faciliter leur émigration économique (poids des Croates d'Herzégovine dans la diaspora croate ou 
des Musulmans du Sandžak dans la diaspora musulmane), ou encore s'emparer des activités liées à la criminalité organisée.

Tentant de dresser le "profil-type" du combattant du Mouvement tchetnik serbe, le quotidien serbe indépendant Borba ("Combat") écrit que celui-ci

a entre 30 et 35 ans, est diplômé de l'enseignement secondaire technique, a un emploi, a au moins un enfant et un mariage raté derrière lui. La majorité des volontaires est d'origine rurale, mais réside dans une ville moyenne (le plus souvent, Smederevo ou Leskovac). Un volontaire sur cinq a eu de sérieux problèmes avec la justice (peines de prison fermes ou avec sursis), et certains sont arrivés au front directement après leur sortie de prison. D'après des témoignages non confirmés, on offre aux condamnés des remises de peine s'ils acceptent de rejoindre les volontaires ${ }^{23}$.

L'importance des solidarités claniques dans les mobilisations miliciennes s'explique donc moins par leur enracinement dans les hauts villages du karst dinarique que par leur redéploiement dans les interstices d'une modernisation mal maîtrisée et par leur extension au "village global". La meilleure illustration de cette réalité reste le rôle des Herzégoviniens de la diaspora dans le financement des forces armées croates. Cette réalité ambiguë des milices, à mi-chemin entre village et "village global", se retrouve d'ailleurs dans leur production symbolique. Ainsi, les "unités spéciales" de la police de la "République serbe de Krajina" se surnomment "Knindjas", association de "Knin", nom de la capitale de cet État auto-proclamé, et des "tortues ninjas", personnages d'un célèbre dessin animé japonais. De façon similaire, le nom de "Bérets verts" fait tout à la fois allusion au couvre-chef distinctif des Musulmans bosniaques (le béret, adopté après l'interdiction du fes en 1950) et à certaines unités d'élite de l'armée américaine. 


\section{Le « folklore » milicien comme invention de la tradition}

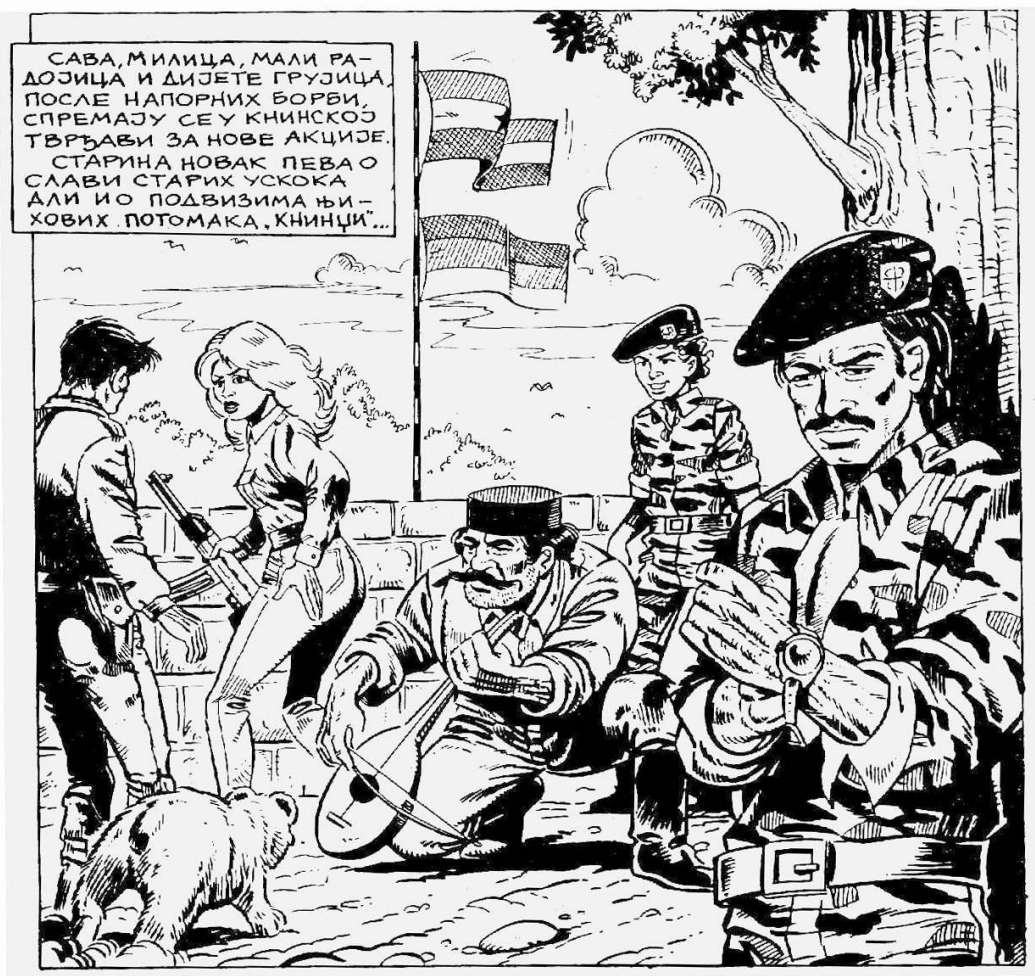

(« Les Knindjas »), Belgrade (1991) : la gusla, signe d'héroïsme

Alors que la plupart des milices politico-mafieuses et des "unités spéciales" affectionnent un style directement repris des films de guerre américains (ray-bans, teeshirts sans manches et guirlande de balles autour du cou), et que les "brigades musulmanes" vont chercher leur inspiration vestimentaire chez les pasdaran iraniens (bandeaux verts portant des versets du Coran noués autour du front), les milices serbes se revendiquant du mouvement tchetnik revêtent plus volontiers la panoplie du parfait hajduk (barbe non taillée, cheveux longs, toque de fourrure). Plus largement, les mobilisations nationalistes s'accompagnent en Serbie d'une remise au goût du jour de la chanson épique, et d'une "folklorisation" généralisée du discours politique ${ }^{24}$ : alors que V. Šešelj décerne le titre de "vojvoda" à ces lieutenants, Ž. Ražnjatović - Arkan célèbre en costume monténégrin, et sans omettre de tirer quelques coups de feu en l'air, son mariage avec Svetlana Veličković - Ceca, jeune star du "turbo-folk" belgradois.

31 Le recours aux registres symboliques de la montagne reste un des signes distinctifs de certaines milices serbes, même si elle n'est pas leur apanage exclusif (le premier ministre de la défense croate, Sime Đodan, faisant par exemple l'éloge de «l'homme dinarique, courageux, franc, enclin aux attitudes chevaleresques, et non sournoises $»^{25}$ ). Il est d'ailleurs significatif que la gusla, instrument à corde typiquement montagnard servant à accompagner les chants épiques, soit présentée comme un attribut des combattants serbes, dans les bandes dessinées de guerre serbes comme dans les bandes dessinées de guerre bosniaques. Dans un cas, la gusla renvoie à l'héroïsme du combattant en question, dans l'autre, à son primitivisme. 


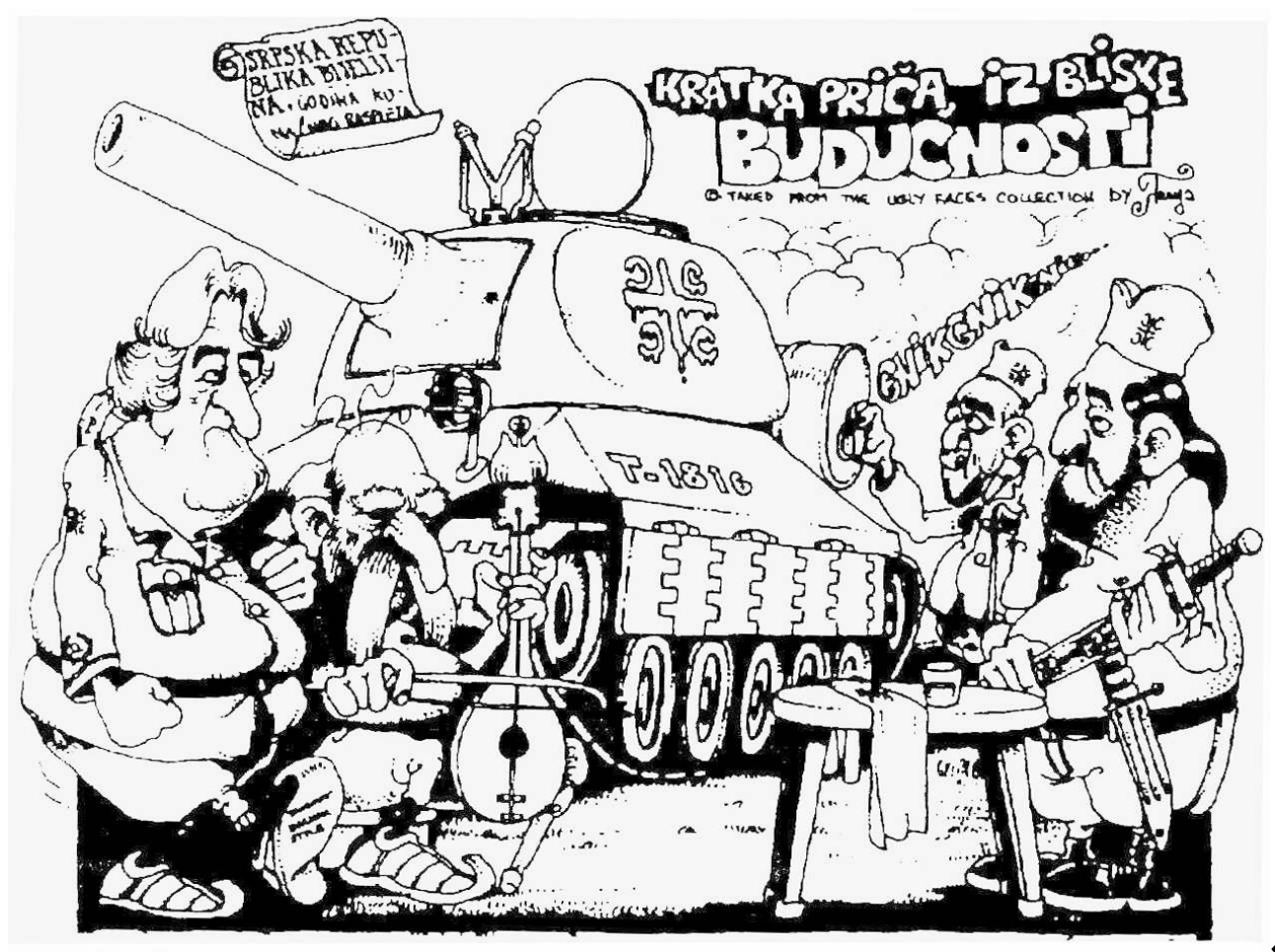

strip art" ("Strip art de guerre »), Lukavac (1994): la gusla, signe de primitivisme

Mais, là encore, une telle "ethnicisation" d'un attribut de la montagne est une reconstruction abusive: la gusla est un instrument utilisé traditionnellement par l'ensemble des populations dinariques, sans distinction d'appartenance ethnique, et la chanson épique ne vient alimenter le "folklore" des milices serbes qu'après un détour par les académies des sciences et les cercles littéraires ou, plus prosaïquement, par les stades de football ${ }^{26}$. Dans la production folklorique des milices également, l'appropriation des figures et des gestes de la montagne représente moins un réveil de la mémoire collective qu'une invention de la tradition, et renvoit aux mêmes constructions idéologiques que les typologies de J. Cvijić ou de D. Tomašić.

\section{Les tchetniks : des hajduk aux auxiliaires de police}

La référence de certaines milices au mouvement tchetnik permet par contre de rattacher les phénomènes miliciens à une dimension spécifique de la modernisation de l'espace yougoslave, à savoir l'émergence de l'État-nation moderne. Tout comme le renouvellement chronique des élites urbaines s'insère dans un processus plus large d'urbanisation, la récurrence des mobilisations de type milicien participe en effet d'un processus ininterrompu et inachevé de modernisation politique.

Les pratiques militaires des Empires ottoman et austro-hongrois, dont certaines évoquent par leur mode de recrutement et de rémunération les milices contemporaines, renvoient à un mode indirect d'exercice du monopole de la violence légitime, caractéristique des systèmes politiques impériaux. À partir du XIX ${ }^{\text {ème }}$ siècle, la crise de ces Empires et l'émergence des États-nations modernes s'accompagnent, dans l'espace yougoslave comme dans l'ensemble des Balkans, d'une recomposition des modes et des sphères d'exercice de ce monopole, rythmée par une série d'insurrections 
nationales et de crises régionales, et dont la Première Guerre mondiale représente en quelque sorte le paroxysme. Or, ces insurrections et ces crises montrent comment les nouveaux États nationaux font à leur tour appel à des formations armées annonciatrices des milices contemporaines, ce dont témoignent en particulier les relations entre l'État serbe, les organisations nationalistes grand-serbes et le mouvement tchetnik.

Né en 1812 d'une insurrection encadrée par d'anciens hajduk, l'État serbe fonde ses premières forces armées sur des milices paysannes, dont les combattants doivent fournir leur uniforme et leur armement personnels. Défaites par une armée ottomane modernisée en 1876, malgré le renfort d'officiers russes pan-slavistes et de volontaires garibaldiens, ces milices paysannes font progressivement place à une véritable armée, encadrée par un corps d'officiers professionnels ${ }^{27}$. L'État serbe continue toutefois de faire appel pour ses campagnes militaires à des formations de volontaires recrutés dans l'ensemble de l'espace yougoslave, et d'entretenir des réseaux insurrectionnels à l'extérieur de ses frontières. Mis en place dans les années 1860 par le ministre de l'intérieur Ilija Garašanin, ces réseaux sont encadrés à partir de 1911 par l'organisation "l'unification ou la mort" (Ujedinjenje ili smrt), plus connue sous le nom de "Main noire" (Crna ruka). Constituée par des officiers de l'armée serbe et commandée par le capitaine Dragutin Dimitrijević - Apis, celle-ci est souvent considérée comme le véritable commanditaire de l'attentat de Sarajevo, commis en juillet 1914 par les membres de l'organisation révolutionnaire "Jeune Bosnie" (Mlada Bosna). Véritable État dans l'État, elle est démantelée en 1917 par le régent Alexandre, avec le soutien d'une organisation d'officiers concurrente, la "Main blanche" (Bela ruka) ${ }^{28}$.

C'est dans cette configuration complexe entre réseaux insurrectionnels, formations de volontaires et organisations nationalistes constituées au sein même de l'armée serbe que se situe le mouvement tchetnik, ensemble de formations armées irrégulières se réclamant du nationalisme grand-serbe. Après l'insurrection de 1903 en Macédoine, l'État serbe encourage en effet la formation de bandes armées sur ce territoire, qualifié par les nationalistes grand-serbes de "Vieille Serbie». Animées par des étudiants nationalistes, d'anciens officiers ou des vojvoda locaux, elles sont constituées sur le modèle des bandes de hajduk (hajdučke čete), et en reprennent la terminologie pour désigner leurs techniques de guérilla (četovanje) et leurs combattants (četnik): le mouvement "tchetnik" est né ${ }^{29}$.

37 Cet emploi de formations tchetniks, agissant derrière les lignes de front, se reproduit lors des guerres balkaniques et de la Première Guerre mondiale. Le terme de "tchetnik" perd alors sa signification sociale d'origine pour prendre une connotation nationale de plus en plus prononcée, montrant ainsi comment le mouvement tchetnik se situe à la charnière entre une violence contre l'État et une violence d'État. Il en va de même des unités de volontaires qui viennent grossir les rangs de l'armée serbe, et dont les membres sont recrutés par les organisations nationalistes agissant sur le territoire des Empires ottoman et austro-hongrois, ou plus simplement attirés par les promesses d'attribution d'une terre et les opportunités de pillage.

Après la création du Royaume des Serbes, Croates et Slovènes en 1918, son armée est constituée à partir du corps des officiers des armées serbe et monténégrine et étroitement contrôlée par la "Main blanche". Par contre, ce premier État yougoslave connaît pendant plusieurs années de sérieuses difficultés pour établir son autorité sur l'ensemble de son territoire. Parmi les populations non-serbes du royaume, le refus de 
la conscription et de l'impôt provoque plusieurs mouvements de révolte armée (jacqueries paysannes en Croatie, soulèvement tribaux au Monténégro, guérilla des kaçaks albanais et des komitadjis macédoniens en "Serbie du sud") ${ }^{30}$. Pour épauler l'armée, l'État yougoslave fait alors de nouveau appel aux formations tchetniks. Organisées en unités de la gendarmerie ou en "garde nationale", celles-ci exercent une répression brutale, souvent accompagnée de pillage ou d'appropriation illégale des terres. Par la suite, les associations d'anciens combattants tchetniks continuent de jouer un rôle d'appoint dans la vie politique du royaume, lors des campagnes électorales ou dans la répression des mouvements revendicatifs.

Cette nouvelle articulation entre l'État yougoslave et le mouvement tchetnik peut être illustrée par la personnalité de Puniša Račić, président de l'“Association des Tchetniks Serbes Petar Mrkonjić pour le Roi et la Patrie" (Udruženje Srpskih Četnika "Petar Mrkonjić" za Kralja i Otadžbinu). Celui-ci, issu du clan monténégrin des Vasojevići, est engagé dans les activités du mouvement tchetnik en Macédoine au moment des guerres balkaniques. Il appartient à la "Main blanche" et participe à ce titre à l'élimination de D. Dimitrijević - Apis en 1917. Après 1918, il organise l'insurrection des tribus catholiques de l'Albanie du nord, avant d'aller réprimer celle des kaçaks albanais en Macédoine. Devenu à cette occasion riche propriétaire terrien, il est élu député en 1927 et se rend célèbre en assassinant en plein Parlement, le 20 juin 1928, le dirigeant du Parti paysan croate, Stjepan Radić.

\section{Violence contre l'État, violence pour l'État}

Le cas "exemplaire" de P. Račić montre comment le mouvement tchetnik passe progressivement d'une activité insurrectionnelle à l'extérieur des frontières de la Serbie à une activité répressive dans celles de la Yougoslavie. Il montre aussi comment cette violence d'État engendre à son tour de nouvelles violences contre l'État, et précipite finalement l'effondrement de l'État yougoslave. L'assassinat de S. Radić, en effet, exacerbe le conflit serbo-croate et conduit, le 6 janvier 1929, à l'instauration d'un régime dictatorial s'appuyant sur l'armée yougoslave, les associations tchetniks et des mouvements de jeunesse organisés sur le modèle fasciste, telles que la Jeunesse nationale serbe (Srpska Nacionalna Omladina - SRNAO). La même année se constituent en Croatie des formations para-militaires locales proches du Parti paysan, appelées "Protection civique" (Građanska zaštita), ainsi que le mouvement des "oustachis", organisation terroriste fondée par le Parti croate du droit, ultra-nationaliste, que dirige Ante Pavelić, et soutenue par l'Italie mussolinienne ${ }^{31}$.

41 Comme le mouvement tchetnik avant lui, le mouvement oustachi s'implante dans les régions dinariques, et en particulier en Herzégovine occidentale, où il organise des attaques de commissariats et de bâtiments officiels en s'appuyant sur des populations encore rétives à la conscription et à l'impôt. Au demeurant, le terme d"'oustachi" (ustaše, sg. : ustaša), signifiant littéralement "insurgé", a une origine similaire à celui de "tchetnik" : avant 1929, il n'a aucune connotation nationale spécifique, et se retrouve même dans certains documents militaires serbes de l'époque des guerres balkaniques pour désigner... les bandes de tchetniks serbes! Il ne prend sa signification contemporaine de nationaliste grand-croate que dans les années trente et quarante, après la création du mouvement oustachi et de l'État indépendant de Croatie (Nezavisna Država Hrvatska - NDH). 
42 En avril 1941 en effet, après l'effondrement de l'armée yougoslave face à l'armée allemande, un État indépendant de Croatie est constitué par les pays de l'Axe, et Ante Pavelić nommé chef (poglavnik) de cet État-satellite. La "protection civique" est alors transformée en système de défense local (domobranstvo), pendant que les groupes de combattants oustachis fournissent les cadres des troupes spéciales du NDH, chargées en particulier de l'élimination des populations serbes. Celles-ci, trouvant refuge dans les régions montagneuses, rejoignent alors un mouvement de résistance tchetnik souvent encadré par d'anciens officiers de l'armée yougoslave. Les rôles se trouvent donc subitement inversés: Andrija Artuković, responsable d'une célèbre attaque de commissariat à Brusani en 1932, devient ministre de l'intérieur du NDH, pendant que Draža Mihailović, ancien colonel de l'armée yougoslave, prend la tête d'un mouvement tchetnik bientôt qualifié de "terroriste", et pourchassé comme tel.

Les basculements entre violence contre l'État et violence d'État ne sont donc pas le fait du seul État serbe. Déjà, pendant les guerres balkaniques, les tchetniks serbes sont confrontés en Macédoine aux komitadji bulgares, aux antar grecs et aux basibozuk ottomans; de même, pendant la Première Guerre mondiale, ils sont combattus en Bosnie-Herzégovine par des Schutzkörper ("corps de protection") recrutés dans les populations locales croates et musulmanes ${ }^{32}$. Pendant la Seconde Guerre mondiale enfin, période de l'histoire où les différents projets stato-nationaux présents dans l'espace yougoslave s'affrontent avec le plus de violence, ce foisonnement de formations armées atteint son paroxysme: aux domobranstvo et aux forces spéciales oustachies du NDH d'une part, aux formations tchetniks organisées autour d'un vojvoda local ou liées à l'état-major de D. Mihailović d'autre part, s'ajoutent en effet une multitude de formations musulmanes locales, sans oublier bien sûr le mouvement des partisans ${ }^{33}$.

En dépit de l'idéologie révolutionnaire qui l'anime, celui-ci s'inscrit dans la continuité des phénomènes de décomposition et de recomposition étatiques qui caractérisent l'histoire de l'espace yougoslave. Son organisation militaire articule d'ailleurs elle aussi deux types de formations (brigades locales et "brigades prolétariennes" mobiles et idéologisées), et son succès final passe par l'intégration en son sein d'éléments entiers des autres formations armées qu'il combat. 50 ans plus tard, l'Armée populaire yougoslave, issue de la militarisation et de l'institutionnalisation de ce mouvement, se trouve à son tour impliquée dans une configuration complexe de violences d'État et de violences contre l'État, et emportée par une nouvelle recomposition des réalités et des légitimités étatiques de l'espace yougoslave.

\section{Récurrence des phénomènes miliciens et inachèvement de l'État}

Historiquement, l'espace yougoslave apparaît donc marqué par des configurations complexes et mouvantes entre violences contre l'État et violences d'État. Ces configurations, à leur tour, renvoient à l'histoire politique de la péninsule balkanique, dans laquelle le processus de constitution de l'État moderne apparaît contradictoire et inachevé : son monopole de la violence légitime reste fragile, ses frontières territoriales et institutionnelles incertaines. Cela est d'autant vrai pour le seul État balkanique qui, après 1918, ne se définisse pas comme un État national, à savoir la Yougoslavie. 

internationalement reconnus, "républiques" auto-proclamées et phénomènes miliciens, montrent bien à quel point, dans l'espace yougoslave, les frontières territoriales et institutionnelles de l'État restent encore mal définies. Cette incertitude est antérieure à l'éclatement de la fédération yougoslave, comme le montrent les rapports entre institutions fédérales et républiques constitutives, entre Armée populaire yougoslave et Défenses Territoriales, ou encore entre services secrets et criminalité organisée, dans le cas paradigmatique de Ž. Ražnjatović - Arkan. Elle survit à la consolidation des États nationaux et à la militarisation de leurs forces armées, comme le signale la persistance des phénomènes miliciens en Serbie et en Croatie, ou celle, dans le cas spécifique de la Bosnie-Herzégovine, de l'articulation entre brigades locales d'une part, brigades mobiles et "unités spéciales" d'autre part, rappelant celle entre milices locales et milices politico-mafieuses dont elles sont généralement issues. Dans ce contexte, les phénomènes miliciens apparaissent comme un instrument indispensable dans la recomposition des réalités et des légitimités étatiques de l'espace yougoslave, et comme une manifestation durable de son inachèvement.

De toute évidence, ces phénomènes miliciens s'appuient sur certaines pratiques présentes dans les sociétés de l'espace yougoslave, et dans leurs populations dinariques en premier lieu. À la force des solidarités claniques s'ajoutent ici certaines pratiques de la violence telles que la possession et l'exhibition individuelles des armes ou la persistance des codes de l'honneur et du sang. Faire des phénomènes miliciens le signe d'un "retour des sociétés" n'en constituerait pas moins un renversement de leur réalité et de leur finalité. Dans les guerres balkaniques comme dans les récents conflits yougoslaves, les formations armées de type milicien viennent d'abord compenser la difficulté des États à conduire leurs populations dans la guerre : c'est après l'échec des mobilisations de l'automne 1991 en Serbie que l'Armée Populaire Yougoslave encourage la constitution des milices de Ž. Ražnjatović - Arkan et de V. Šešelj.

Dans l'inadéquation entre réalités des sociétés balkaniques et constructions statonationales qui caractérisent l'espace yougoslave, les phénomènes miliciens se situent clairement du côté de l'État, provoquant d'abord les tensions et les mobilisations miliciennes locales, arrachant ensuite les combattants locaux à leurs terroirs au nom de la défense d'un territoire, chassant enfin les populations dont l'identité ethnique ne correspond pas à celle du dit territoire. Outil privilégié de construction des Étatsnations, les phénomènes miliciens sont renvoyés par les idéologies nationalistes aux profondeurs barbares des sociétés. Ce renversement, du reste, est peut-être logique, voire indispensable. Car, dans un espace yougoslave où l'État ne parvient pas à imposer durablement ni le monopole, ni la légitimité de sa propre violence, il reste à savoir si les phénomènes miliciens en constituent le dévoiement, ou le dévoilement.

\section{NOTES}

1. Straus (Ivan), Sarajevo, l'architecte et les barbares, Paris : Linteau, 1994. 
2. L'expression raja provient du terme ra'ya (pl. : re'aya) qui, dans l'ordre politique ottoman, désigne les producteurs, les sujets, en opposition aux asker, militaires et membres de l'appareil d'État. À l'origine, le terme raja n'a donc pas de connotation spécifiquement urbaine : dans la Bosnie ottomane, il désigne même avant tout les populations rurales et chrétiennes travaillant les terres des beg et aga musulmans. Le terme papak (pl. : papci) désigne quant à lui les sabots des animaux d'élevage (onglons). Dans la bouche des Sarajéviens, les paysans sont donc assimilés à leur bétail.

3. Le terme de Balije, utilisé de façon péjorative pour les Musulmans bosniaques, désigne à l'origine des populations nomades musulmanes d'Herzégovine orientale ; de même, les Valaques sont une population nomade orthodoxe, de langue aroumaine, utilisée comme troupes auxiliaires lors de l'expansion de l'Empire ottoman, et dont serait originaire une partie de la population serbe bosniaque.

4. Les Alpes dinariques constituent l'ensemble montagneux qui s'étend des Alpes slovènes aux Rhodopes bulgares, et recouvrent en particulier les régions de l'arrière-pays dalmate (Krajina croate), de l'Herzégovine, du Monténégro et du Sandžak.

5. Cvijić (Jovan), La péninsule balkanique, Paris: Armand Colin, 1918. Pour une présentation récente et synthétique de cette articulation entre ville, plaine et montagne, voir Prevelakis (Georges), Les Balkans. Cultures et géopolitique, Paris : Nathan, 1994.

6. Tomašić (Dinko), «Plemenska kultura i njeni današnji ostaci », paru dans Mjesečnik, 62 (1), janvier 1936, et reproduit dans "Sociologija Dinka Tomašića », Društvena istraživanja, 2 (6), novembre 1992.

7. Tomašić (Dinko), Personality and Culture in East European Politics, New York : Georges Stewart Pub., 1948.

8. Stulhofer (Aleksandar), «Predviđanje rata? Etnosociologija Dinka Tomašića », Društvena istraživanja, 2 (2), mars 1992.

9. Društvena istraživanja, « Sociologija Dinka Tomašića », 2, 1992.

10. Meštrović (Stjepan), Letica (Slaven), Goreta (Miroslav), Habits of the Balkan Heart. Social Character and the Fall of Communism, College Station : Texas A\&M University Press, 1993.

11. Bakić-Hayden (Milica), Hayden (Robert M.), "Orientalist Variations on the Theme "Balkans": Symbolic Geography in recent Yugoslav Cultural Politics », Slavic Review, 51 (1), printemps 1992.

12. Sur l'insertion du pélerinage de Međugorje dans la culture clanique de l'Herzégovine occidentale, voir les travaux de Mart Baxt, et en particulier Baxt (Mart), « The Saints of Gomila : Belief and Blood Vengeance in a Yugoslav Peasant Society », Ethnologia European, (22), printemps 1992.

13. Ziga (J.), Islamska civilizacija $i$ bosanski Muslimani, Sarajevo: Vijeće Kongresa Bosanskomuslimanskih Intelektualaca, 1994.

14. Borogovac (M.), «Kulturni kanibalizam», Muslimanski glas, 07/02/92. Le terme čitluk ou čiftluk (turc: ciftlik) désigne en Bosnie ottomane les propriétés foncières des beg et des aga musulmans.

15. Tomašić (Dinko), op. cit.

16. Sur la pensée d'Ibn Khaldun et ses interprétations contemporaines, voir en particulier les ouvrages de Aziz Al-Azmeh (Al-Azmeh (Aziz), Ibn Khaldun in Modern Scholarship, London : Third World Center, 1981, et Ibn Khaldun. An Essay in Reinterpretation, London : Frank Cass, 1982).

17. Al-Azmeh (Aziz), Ibn Khaldun. An Essay (op.cit.).

18. Sur la place des Slaves du sud dans l'organisation militaire ottomane, voir Majer (H. G.), "Albaner und Bosner in der osmanischen Armee. Ein Faktor der Reichsintegration im XVII. und XVIII. Jahrhundert », in Grothusen (K.), ed., Jugoslawien. Integrationsprobleme in Geschichte und Gegenwart, Göttingen : Vandenhoeck \& Ruprecht, 1986, ainsi que Suceska (Avdo), « The Position 
of the Bosnian Moslems in the Ottoman State », International Journal of Turkish Studies, 1 (2), automne 1980.

19. Sur les Militärgrenzen, voir Rothenberg (G.), "The Habsburg Military Border System », in Király (Béla), Rothenberg (G.), eds., War and Society in East Central Europe (18th and 19th Centuries), New York : Columbia University Press, 1979.

20. Tomašić (Dinko),art.cit.

21. Tomašić (Dinko), op cit., p. 115.

22. Ce phénomène de "retraditionnalisation" de la société yougoslave a été mentionné par le sociologue croate Josip Županov dès 1970, et étudié dans un ouvrage de référence consacré à « l'autogestion et le pouvoir social » (Županov (Josip), Samoupravljanje i društvena moć, Zagreb : Globus, 1985). Sur la reproduction des solidarités familiales et claniques, voir également Canapa (Marie-Paule), "Traditions communautaires paysannes et socialisme en Yougoslavie ", Revue d'études comparatives Est-Ouest, 21 (2), juin 1990.

23. Katić (G.), Mikuljanac (M.), «Kako su formirane dobrovoljačke jedinice u Srbiji ? », Borba, 20-21/11/93.

24. L'ethnologue serbe Ivan Čolović a consacré de nombreux travaux à cette question de la "folklorisation" du discours politique en Serbie (Čolović (Ivan), Bordel ratnika, Beograd : XX. vek, 1993, et Pucanje od zdravlja, Beograd: Krug, 1995). Parmi les textes d'Ivan Čolović traduits en français, voir plus particulièrement Čolović (Ivan), «Le folklore et la politique : une affaire moderne », in Morokvašić (Mirjana), dir., "Yougoslavie: logiques de l'exclusion », Peuples méditerranéens, (61), octobre-décembre 1992.

25. Batušić (Z.), « Dinarski superčovjek », Danas, 19/05/92.

26. Sur le rôle du football dans les mobilisations nationalistes et miliciennes, voir le dossier de la revue indépendante croate Erasmus, (n 10, février 1995), et en particulier l'article d'Ivan Čolović consacré aux liens entre la Garde des volontaires serbes et les supporters de l'"Étoile rouge", les delije (nom dérivé de celui de certaines formations militaires auxiliaires... ottomanes !).

27. Voir Király (Béla), Stokes (Gale), eds., Insurrections, Wars and the Eastern Crisis in the 1870s, New York : Columnia University Press, 1985.

28. Voir MacKenzie (David), «Serbian Nationalist and Military Organisations and the Piedmont idea (1844-1914 », East European Quarterly, 16 (3), septembre 1982.

29. Sur l'histoire du mouvement tchetnik, voir en particulier Tomasevic (Jozo), The Chetniks, Stanford: Stanford University Press, 1975, ainsi que Sehić (Nusret), Četnički pokret u Bosni $i$ Hercegovini (1918-1941), Sarajevo : ANUBiH, 1971.

30. Voir Banac (Ivo), The National Question in Yugoslavia, Ithaca-London : Cornell University Press, 1984.

31. Sur le mouvement oustachi, voir Krizman (Bogdan), Ante Pavelić i Ustaše, Zagreb : Globus, 1983.

32. Sur les guerres balkaniques, voir Király (Béla K.), Djordjevic (Dimitrije), eds., East Central European Society and the Balkan Wars, New-York : Columbia University Press, 1987.

33. Sur la Deuxième Guerre mondiale dans l'espace yougoslave, voir en particulier Roberts (W.), Tito, Mihailovic and the Allies 1941-1945, Durham: University of New Brunswick, 1973. Sur les formations musulmanes, voir Redžić (Enver), Muslimansko automaštvo i 13. SS divizija, Sarajevo : Svjetlost, 1987, ainsi que Jahić (A.), Muslimanske formacije tuzlanskog kraja u drugom svjetskom ratu, Tuzla : Bosnoljublje, 1995. 


\section{RÉSUMÉS}

Alors qu'une partie des journalistes et des intellectuels ont refusé de conceptualiser les conflits yougoslaves comme atavisme balkanique, ils ont tout de même cédé à les présenter en tant que « revanche des campagnes", ce qui ne décrit pas non plus la réalité de ces conflits, mais est utile aux acteurs politiques : l'idée d'une ville « civilisée » opposée à une campagne « barbare " nourrit les haines de classe et les discours guerriers.

Si les rapports entre ville et campagne occupent une place centrale dans ces conflits comme dans le discours de leurs protagonistes, encore faut-il savoir quelle est la part de la réalité et la part du mythe, quelle est la véritable signification de ces rapports, et se demander en particulier ce qu'ils révèlent (ou dissimulent) sur les rapports entre milices, État et société dans l'espace yougoslave. L'importance des solidarités claniques dans les mobilisations miliciennes s'explique moins par leur enracinement dans les hauts villages du karst dinarique que par leur redéploiement dans les interstices d'une modernisation mal maîtrisée et par leur extension au « village global ».

Dans les guerres balkaniques comme dans les récents conflits yougoslaves, les formations armées de type milicien viennent d'abord compenser la difficulté des États à conduire leurs populations dans la guerre.

Although a part of reporters and intellectuals have refused to conceptualise Yugoslavian conflicts as an atavism from the Balkans, they accepted to interpret them as a «revenge from countryside", which does not, as the first, describe the reality of these conflicts. But this argumentation is useful for political actors: the idea of a "civilised " town vs. a "barbaric " countryside helps to nurture the class's hate and war discourses.

If the relations between city and countryside fill a central place in the conflicts as well as in the discourses of their protagonists, we have to know what is the part of truth and the one of myth, what signification they have, what the reveal or, on the contrary, hide on the relations between militias, state and community in the Yugoslavian space. The importance of clanic solidarity in the mobilisation of militias is much more explained by its new extension in the interstices of a modernisation badly controlled and to the " global village " than by its rooting in the villages in the dinaric karst's mountains.

In the Balkan wars, as well as in the recent Yugoslavian conflicts, the armed units, like militias, serve, first of all, to offset states' difficulties to lead their populations into war.

\section{AUTEUR}

\section{XAVIER BOUGAREL}

X. Bougarel est doctorant en science politique à l'IEP de Paris. 